\title{
Pancreatic cancer metastasis to the rectum detected on colonoscopy
}

An 87-year-old man was referred to our institution for further investigation of bloody stool. The patient had undergone partial gastrectomy for gastric cancer about 40 years earlier and right lower lobectomy for well-differentiated squamous cell carcinoma in the right lung about 10 years earlier. He had been taking medication for primary hypertension and benign prostatic hypertrophy for 9 years. Colonoscopic examinations revealed reddish mucosa with convergence of mucosal folds in the rectum ( $\bullet$ Figs. 1, 2). Histological examination (hematoxylin and eosin staining) of the biopsy specimens revealed adenocarcinoma ( $\bullet$ Fig. 3 ). Immunohistologically, the specimens were positive for cytokeratin 7 and negative for cytokeratin 20 and prostate-specific antigen [1]. An abdominal computed tomography scan showed a hypovascular tumor in the tail of the pancreas, multiple metastatic tumors in the liver, and multiple nodules in the omentum ( $\bullet$ Fig. 4). A hypovascular mass was also observed adjacent to the rectum and was considered to be one of the nodes of peritoneal dissemination ( Fig. 5). Upper gastrointestinal endoscopy revealed no tumor. Based on these findings, we diagnosed the patient as having pancreatic cancer disseminated to the rectum and he was given best supportive care possible.

Patients with pancreatic cancer are often asymptomatic for long time before the cancer manifests with features related to local and distant spread. It has been reported that carcinomas of the body and/ or tail of pancreas are more frequently characterized by transperitoneal as well as hematogeneous dissemination than carcinoma of the head of pancreas [2,3]. Although pancreatic cancer often metastasizes to the stomach or duodenum, reports of pararectal metastasis are scarce [4]. In addition, immunohistochemical staining of biopsy specimens for cytokeratin 7 and 20 can be helpful in diagnosing unusual metastasis of pancreatic cancer $[1,4]$.

Endoscopy_UCTN_Code_CCL_1AD_2AC

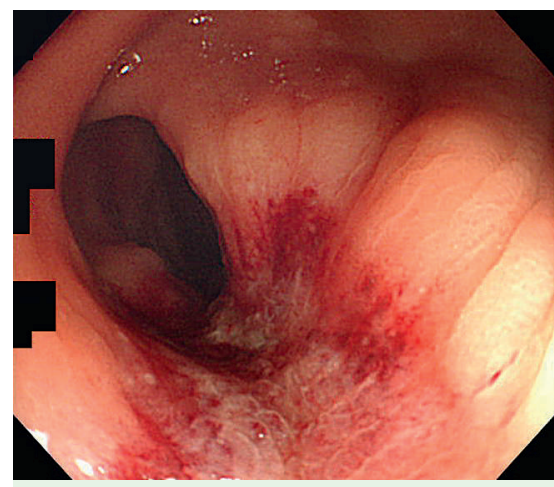

Fig. 1 Colonoscopic examination showing reddish, coarse mucosa in the rectum.

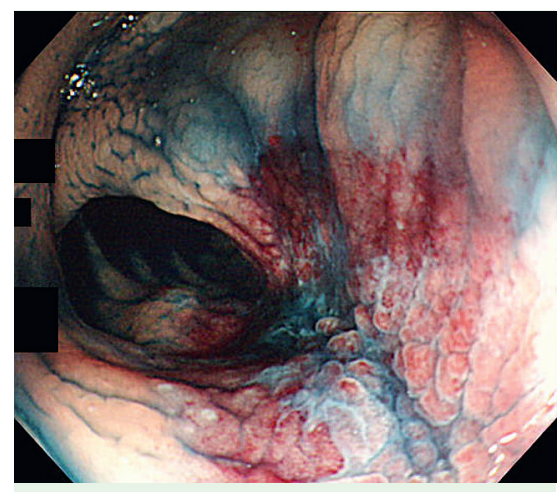

Fig. 2 Colonoscopy with indigo carmine clearly showing mucosal fold convergence in the rectum.

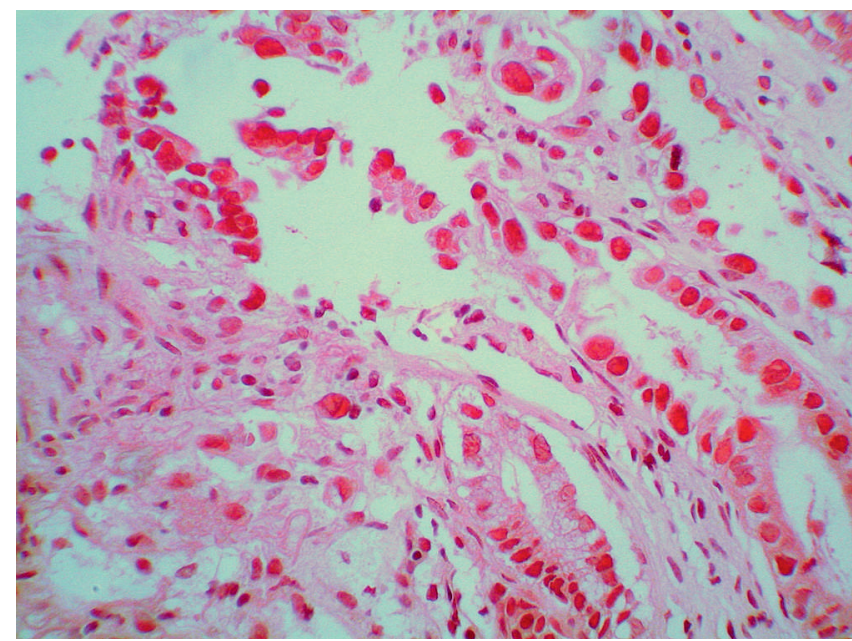

Fig. 3 Histological specimen showing adenocarcinoma.

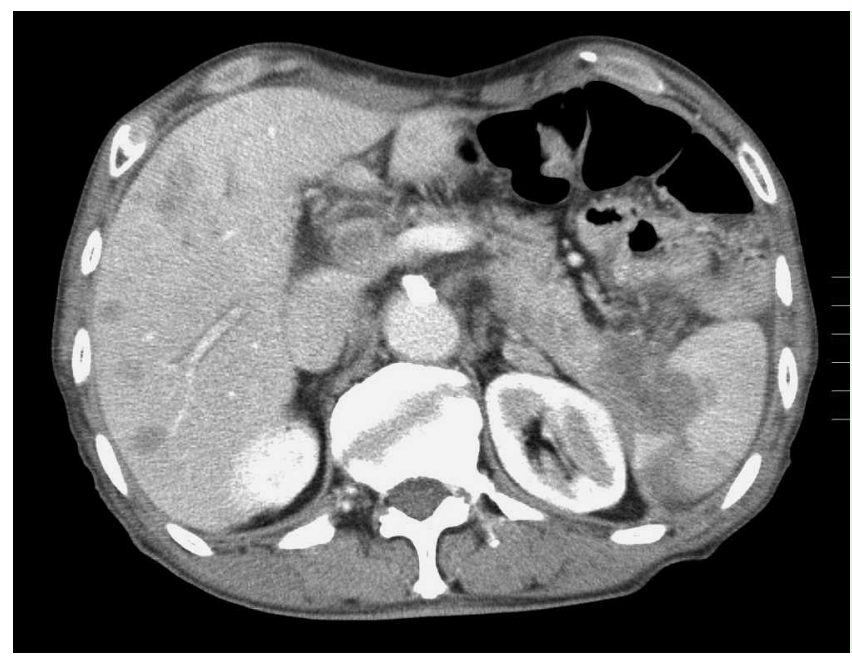

Fig. 4 Abdominal computed tomography scan showing a hypovascular tumor in the tail of the pancreas and multiple metastases in the liver. 


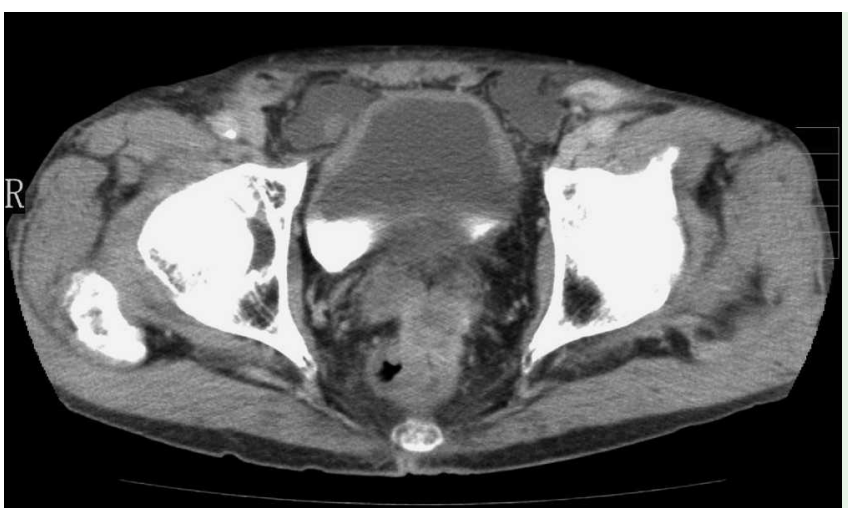

Fig. 5 Abdominal computed tomography scan showing the hypovascular tumor has disseminated to the rectum.

H. Fukatsu ${ }^{1}$, Y. Nagahara ${ }^{1}$, K. Ishiki ${ }^{1}$, M. Iwamura², F. Hamada ${ }^{2}$

1 Department of Gastroenterology, Nihon Nippon Fukuyama Hospital, Fukuyama, Japan

2 Department of Surgery, Nihon Nippon Fukuyama Hospital, Fukuyama, Japan

\section{References}

1 Tot $T$. Adenocarcinomas metastatic to the liver: the value of cytokeratins 20 and 7 in the search for unknown primary tumors Cancer 1999; 85: 171 - 177

2 Mao C, Domenico DR, Kim K et al. Observations on the developmental patterns and the consequences of pancreatic exocrine adenocarcinoma. Findings of 154 autopsies. Arch Surg 1995; 130: 125-134
3 Kamisawa T, Isawa T, Koike $M$ et al. Hematogenous metastases of pancreatic ductal carcinoma. Pancreas 1995; 11: 345-349

4 Bandyopadhyay D, Kapadia CR, Da Costa PE. Pancreatic carcinoma: report of two cases presenting with unusual metastases. Indian J Gastroenterol 2005; 24: 75 - 76

\section{Bibliography}

DOI $10.1055 / \mathrm{s}-0029-1214732$

Endoscopy 2009; 41: E167-E168

(c) Georg Thieme Verlag KG Stuttgart · New York . ISSN 0013-726X

Corresponding author H. Fukatsu, MD, FACP

Department of Gastroenterology Nihon Nippon Fukuyama Hospital 1844 Tsunoshita Daimoncho Fukuyama 721-0927

Japan

Fax: +81-84-945-3564

fukatsu-gi@umin.ac.jp 